\title{
Drohende Frühgeburt: Liegen Schwangere zu kurz?
}

Frühgeburten sind ein großes Problem in der modernen Geburtshilfe, da sie zu einer hohen perinatalen Morbidität und Mortalität führen. Im Jahr 2015 lag die Frühgeburtenrate in Deutschland bei 8,55 Prozent [1]. Bei kurzer Zervix und/oder vorzeitiger Wehentätigkeit wird den Schwangeren als flankierende Maßnahme häufig Bettruhe verordnet. Diese Praxis basiert auf der Beobachtung, dass starke physische Anstrengung zu vorzeitiger Wehentätigkeit führen können. Körperliche Schonung und Bettruhe sind deshalb die am häufigsten getroffenen Anordnungen in der Geburtshilfe. Fast alle Frauenärzte haben sie ihren Patientinnen bereits empfohlen [2]. Ungefähr $18 \%$ der Schwangeren erhalten im Verlauf ihrer Gravidität den Hinweis, sich körperlich zu schonen [3]. Während sich Bettruhe für eine Vielzahl von Indikationen, wie vaginale Blutungen, vorzeitiger Blasensprung, fetale Wachstumsrestriktion und schwangerschaftsbedingte $\mathrm{Hy}$ pertonie anbieten kann, soll im Folgenden nur der Stellenwert von Bettruhe bei drohender Frühgeburt kritisch betrachtet werden.

Ende der 2000er-Jahre gab es 2 Befragungen von Geburtshelfern in Nordamerika zum Thema Bettruhe bei verkürzter Zervixlänge. In Kanada verordneten Geburtshelfer, Allgemeinärzte und Hebammen in jeweils $72,3,77,2$ und $67,1 \%$ der Fälle von drohender Frühgeburt körperliche Schonung [4]. Bei einer entsprechenden Umfrage unter den Mitgliedern der SMFM (Society of Maternal-Fetal Medicine, USA) gaben mehr als $80 \%$ der Geburtshelfer und Perinatalmediziner an, Bettruhe als therapeutische Maßnahme einzusetzen [5]. Viele Hebammen und Frauenärzte glauben weiterhin, dass körperliche Inaktivität ohne negative Folgen für ihre Patientinnen bleibt und dass im Umkehrschluss körperliche Aktivität zu uteriner Kontraktilität, Verkürzung der Zervix und Erweiterung des inneren Muttermunds führt.

- Tab. 1 Einschränkung der körperlichen Aktivität nach [2].

\begin{tabular}{|l|l|}
\hline Definition & Art der Aktivitätsrestriktion \\
\hline leicht & $\begin{array}{l}\text { 1 h oder weniger kontinuierliche Ruhe im Bett oder in sitzender Position } \\
\text { während des Tages. Kein Heben von Gewicht von mehr als } 4,5 \mathrm{~kg} .\end{array}$ \\
\hline moderat & $\begin{array}{l}\text { Mehr als } 1 \mathrm{~h}, \text { aber weniger als } 8 \mathrm{~h} \text { kontinuierliche Ruhe während des Tages, } \\
\text { keine Haushaltsaufgaben und kein Heben von Gewichten. Besuche beim Arzt } \\
\text { sind erlaubt. }\end{array}$ \\
\hline streng & $\begin{array}{l}\text { Aufenthalt im Haus außer zu Arztbesuchen. Kontinuierliche Ruhe im Sitzen oder } \\
\text { im Liegen während des Tages. Keine Haushaltsaufgaben und kein Heben von } \\
\text { Gewichten. }\end{array}$ \\
\hline
\end{tabular}

Leider gibt es bisher keine standardisierte Definition von Bettruhe. Diese ist je nach Quelle definiert als Bettruhe 3-mal täglich für 1 Stunde [6], Bettruhe für 22-23 Stunden am Tag [5] und kontinuierliche Bettruhe für 24 Stunden mit Ausnahme von Toilettengängen [7]. Diese Inkonsistenzen erschweren den Vergleich mehrerer Studien miteinander. Sciscione schlug als Lösung die Einteilung in leichte, moderate und strikte körperliche Schonung vor ( $\triangleright$ Tab. 1 ), um für zukünftige Untersuchungen eine reproduzierbare Einteilung der körperlichen Schonung zu schaffen [2].

\section{Bettruhe bei Einlings- schwangerschaft mit drohender Frühgeburt}

Eine Cochrane-Übersicht aus dem Jahr 2015 fand nur 2 Publikationen, welche die Einschlusskriterien einer guten wissenschaftlichen Untersuchung erfüllten [68]. Für die Metaanalyse kamen nur die Ergebnisse von Hobel et al. in Betracht, da die andere Studie von Elliott et al. auch eine kleine Anzahl von Mehrlingen mit einbezog [8]. Hobel et al. führten eine randomisierte Studie an 8 Krankenhäusern in der Region von Los Angeles durch. Studienteilnehmerinnen waren Hochrisiko- schwangere, die nach einem Risiko-Score evaluiert wurden. Die Interventionsgruppe umfasste 1774 , die Kontrollgruppe 880 Schwangere. 432 Schwangeren wurde Bettruhe zu Hause verordnet, 834 Schwangere erhielten entweder ein Placebo $(n=412)$ oder keine Intervention $(n=422)$. Die Frühgeburtenrate unterhalb von 37 Schwangerschaftswochen war in beiden Gruppen ähnlich (7,9\% in der Interventions-, $8,5 \%$ in der Kontrollgruppe, relatives Risiko 0,92, 95\%-KI 0,62-1,37) und damit statistisch nicht signifikant verschieden [8].

\section{Bettruhe bei Mehrlings- schwangerschaft mit drohender Frühgeburt}

Crowther und Han führten eine Metaanalyse von 7 randomisierten Studien an Schwangeren mit Mehrlingen durch [9]. Die Probandinnen erhielten entweder Bettruhe im Krankenhaus oder wurden erst bei Eintritt von Komplikationen dorthin überwiesen. Routinemäßige Bettruhe senkte weder das Risiko einer Frühgeburt noch die perinatale Mortalität. Während in beiden Studienarmen das Risiko von sehr niedrigem kindlichen Geburtsgewicht $(<1500 \mathrm{~g})$ gleich war, gab es in der Gruppe der hospitalisierten Schwangeren einen 
Trend zu einer niedrigeren Anzahl von Neugeborenen mit einem Gewicht von $<2500 \mathrm{~g}$ (relatives Risiko 0,92, 95\%-KI $0,85-1,00)$. Alle anderen maternalen und neonatalen Outcome-Parameter waren gleich [9].

\section{Negative Auswirkungen von prolongierter Bettruhe}

Obwohl evidenzbasierte Daten Fehlanzeige sind, gibt es eine Reihe von negativen körperlichen und psychologischen Auswirkungen, die eine prolongierte Bettruhe während der Schwangerschaft nach sich ziehen kann. Neben dem Verlust an Knochen- und Muskelmasse und einer erhöhten Rate an Thromboembolien sind weitere Risiken in der Infobox beschrieben [10-14].

KÖRPERLICHE UND PSYCHO-
LOGISCHE AUSWIRKUNG VON
BETTRUHE IN DER SCHWAN-
GERSCHAFT NACH [14]
- kleineres Lungenvolumen,
- Atemnot
- Obstipation
- Insulinresistenz
- Schwindel
- Insomnia, Müdigkeit
- Langeweile
- Konzentrationsschwierigkeiten
- Stress in der Familie
- Depressionen
- Einkommensverlust

\section{FAZIT}

Es gibt keine Daten, die für eine Bettruhe zu Hause/im Krankenhaus sprechen. Auch wenn Bettruhe sehr oft verordnet wird, fehlt jede Evidenz, dass diese Maßnahme von Nutzen ist. Die betreuenden Frauenärzte sollten vor dem Hintergrund der potenziellen Nachteile einer Bettruhe für die schwangere Mutter und der Kosten für das Gesundheitssystem das Für und Wider einer Bettruhe mit der Schwangeren erörtern. Gut konzipierte Studien sind notwendig, um den Stellenwert einer Bettruhe zur Vermeidung einer Frühgeburt prospektiv zu untersuchen. Erst wenn die Vorteile einer Bettruhe belegt sind, sollte diese routinemäßig empfohlen werden. Denn auch hier gilt der Grundsatz: Primum non nocere.

\section{Interessenkonflikt}

Die Autoren geben an, dass kein Interessenkonflikt besteht.

\section{Korrespondenzadresse}

Prof. Dr. Ralf L. Schild, MA FRCOG

Diakovere Krankenhaus gGmbH

Perinatalzentrum Hannover

Schwemannstraße 17-31

30159 Hannover

ralf.schild@diakovere.de

\section{Literatur}

[1] Gesundheitswesen IQTIG. Bundesauswertung zum Erfassungsjahr 2015 Geburtshilfe. 2016. Online: https://iqtig.org/downloads/ ergebnisse/qidb/2015/2016-05-25/QIDB_ 2015_INDIREKT_PDF/QIDB_2015_indirekte _Leistungsbereiche/BuAw_2015_INDIREKT/ bu_Gesamt_16N1-GEBH_2015.pdf; Stand: 03.05.2017

[2] Sciscione AC. Maternal activity restriction and the prevention of preterm birth. Am J Obstet Gynecol 2010; 202: 232.e1-232.5

[3] Goldenberg RL, Cliver SP, Bronstein J et al. Bed rest in pregnancy. Obstet Gynecol 1994; 84: 131-136
[4] Sprague AE, O’Brien B, Newburn-Cook C et al. Bed rest and activity restriction for women at risk for preterm birth: a survey of Canadian prenatal care providers. J Obstet Gynaecol Can 2008; 30: 317-326

[5] Fox NS, Gelber SE, Kalish RB et al. The recommendation for bed rest in the setting of arrested preterm labor and premature rupture of membranes. Am J Obstet Gynecol 2009; 200: 165.e1-165.e6

[6] Hobel C], Ross MG, Bemis RL et al. The West Los Angeles Preterm Birth Prevention Project. I. Program impact on high-risk women. Am J Obstet Gynecol 1994; 170: 54-62

[7] Elliott JP, Miller HS, Coleman S et al. A randomized multicenter study to determine the efficacy of activity restriction for preterm labor management in patients testing negative for fetal fibronectin. J Perinatol 2005; 25: 626-630

[8] Sosa CG, Althabe F, Belizan JM et al. Bed rest in singleton pregnancies for preventing preterm birth. Cochrane Database Syst Rev 2015; (3): CD003581

[9] Crowther CA, Han S. Hospitalisation and bed rest for multiple pregnancy. Cochrane Database Syst Rev 2010; (7): CD000110

[10] Kaji T, Yasui T, Suto M et al. Effect of bed rest during pregnancy on bone turnover markers in pregnant and postpartum women. Bone 2007; 40: 1088-1094

[11] Promislow JH, Hertz-Picciotto I, Schramm M et al. Bed rest and other determinants of bone loss during pregnancy. Am J Obstet Gynecol 2004; 191: 1077-1083

[12] Maloni JA, Schneider BS. Inactivity: symptoms associated with gastrocnemius muscle disuse during pregnancy. AACN Clin Issues 2002; 13: 248-262

[13] Kovacevich G], Gaich SA, Lavin JP et al. The prevalence of thromboembolic events among women with extended bed rest prescribed as part of the treatment for premature labor or preterm premature rupture of membranes. Am J Obstet Gynecol 2000; 182: 1089-1092

[14] McCarty-Singleton S, Sciscione AC. Maternal activity restriction in pregnancy and the prevention of preterm birth: an evidence-based review. Clin Obstet Gynecol 2014; 57: 616627

\section{Bibliografie}

DOI https://doi.org/10.1055/s-0043-108641 Geburtsh Frauenheilk 2017; 77: 741-742 @ Georg Thieme Verlag KG Stuttgart · New York । ISSN 0016-5751 